\begin{tabular}{|c|c|c|c|c|c|c|c|c|}
\hline \multicolumn{3}{|c|}{$\begin{array}{l}\text { 2. To: (Receiving Organization) } \\
\text { Distribution }\end{array}$} & \multicolumn{2}{|c|}{ 3. From: (Originating Organization) } & \multicolumn{4}{|c|}{$\begin{array}{r}\text { 4. Related EDT No.: } \\
\text { N/A }\end{array}$} \\
\hline \multicolumn{3}{|c|}{$\begin{array}{l}\text { 5. Proj./Prog./Dept./Div.: } \\
\text { Spent Nuclear Fuel Project }\end{array}$} & \multicolumn{2}{|c|}{$\begin{array}{l}\text { 6. Design Authority/ Design Agent/Cog. } \\
\text { Engr.: } \\
\text { C. Van Katwijk }\end{array}$} & \multicolumn{4}{|c|}{$\begin{array}{l}\text { 7. Purchase Order No:: } \\
\qquad \text { N/A }\end{array}$} \\
\hline \multirow{2}{*}{\multicolumn{5}{|c|}{$\begin{array}{l}\text { 8. Originator Remarks: } \\
\text { N/A }\end{array}$}} & \multicolumn{4}{|c|}{$\begin{array}{l}\text { 9. Equip./Component No.: } \\
\text { N/A }\end{array}$} \\
\hline & & & & & \multicolumn{4}{|c|}{$\begin{array}{l}\text { 10. System/Bldg./Facility: } \\
\text { Spent Nuclear Facility }\end{array}$} \\
\hline \multirow{3}{*}{\multicolumn{5}{|c|}{ 11A. Design Baseline Document? [] Yes [X] No }} & \multicolumn{4}{|c|}{$\begin{array}{c}\text { 12. Major Assm. Dwg. No.: } \\
\text { N/A }\end{array}$} \\
\hline & & & & & \multicolumn{4}{|c|}{$\begin{array}{l}\text { 13. Permit/Permit Application No.: } \\
\text { N/A }\end{array}$} \\
\hline & & & & & \multicolumn{4}{|c|}{$\begin{array}{l}\text { 14. Required Response Date: } \\
\text { N/A }\end{array}$} \\
\hline 15. & \multicolumn{4}{|c|}{ DATA TRANSMITTED } & (F) & (G) & $(\mathrm{H})$ & (I) \\
\hline $\begin{array}{l}\text { (A) } \\
\text { Item } \\
\text { No. }\end{array}$ & (B) Document/Drawing No. & $\begin{array}{l}\text { (C) } \\
\text { Sheet } \\
\text { No. }\end{array}$ & $\begin{array}{l}\text { (D) } \\
\text { Rev. } \\
\text { No. }\end{array}$ & $\begin{array}{l}\text { (E) Title or Description of Data } \\
\text { Transmitted }\end{array}$ & $\begin{array}{l}\text { Approval } \\
\text { Desig- } \\
\text { nator }\end{array}$ & $\begin{array}{l}\text { Reason } \\
\text { for } \\
\text { Trans- } \\
\text { mittal }\end{array}$ & $\begin{array}{c}\text { Origi- } \\
\text { nator } \\
\text { Dispo- } \\
\text { sition }\end{array}$ & $\begin{array}{l}\text { Receiv- } \\
\text { er } \\
\text { Dispo- } \\
\text { sition } \\
\end{array}$ \\
\hline 1 & SNF-3878 & & 0 & $\begin{array}{l}\text { Griswold Tempered Water Flow } \\
\text { Regulator Valves Used as Anti-Siphon } \\
\text { Valves }\end{array}$ & $\begin{array}{l}\text { PF } 8, Q, Q \\
4-5=99\end{array}$ & 2 & 1 & N/A \\
\hline & & & & & & & & \\
\hline & & & & & & & & \\
\hline & & - & & & & & & \\
\hline & & & & & & & & \\
\hline
\end{tabular}

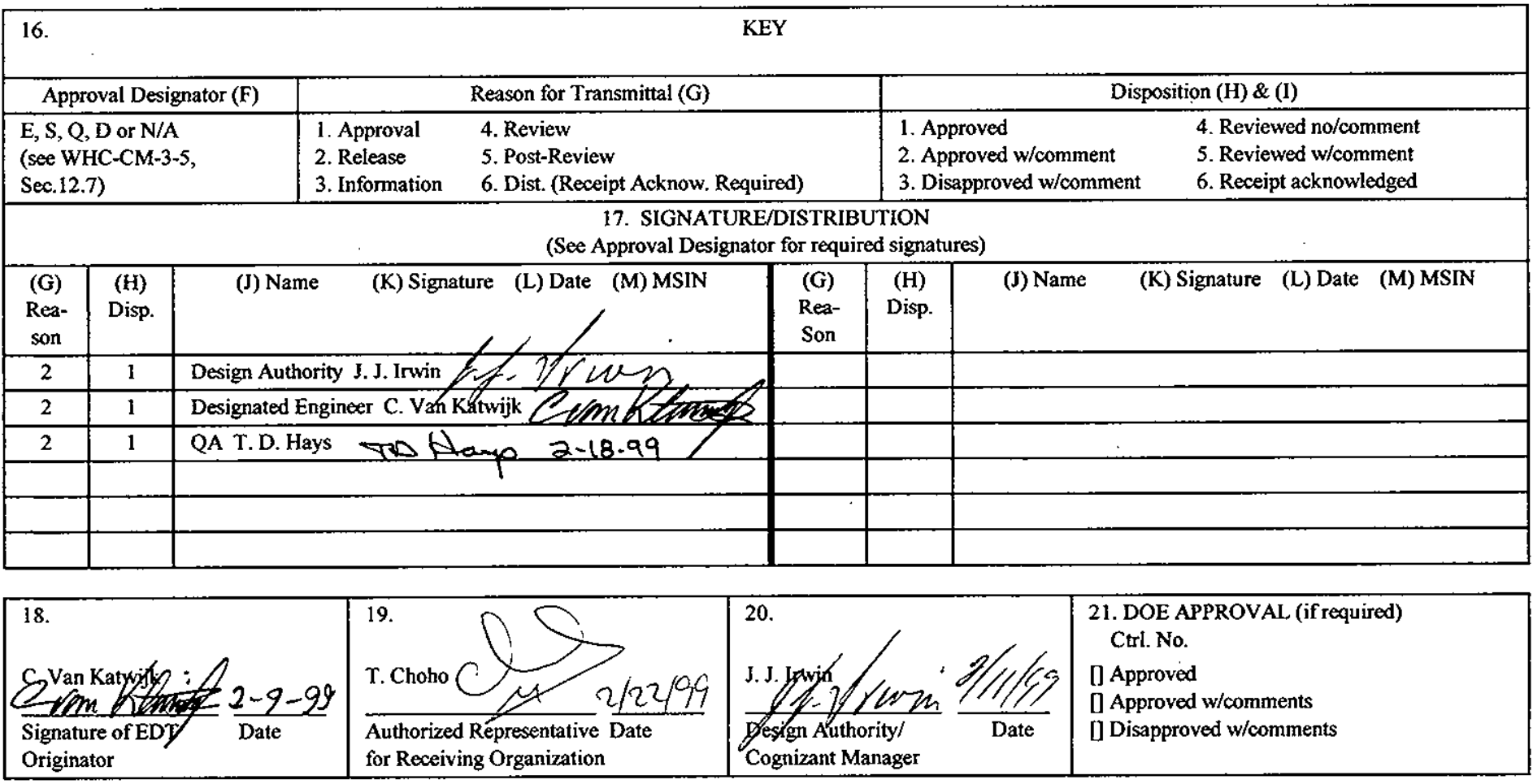




\title{
Griswold Tempered Water Flow Regulator Valves Used as Anti-Siphon Valves
}

\author{
Carl Van Katwijk \\ Numatec Hanford Co, Richland, WA 99352 \\ U.S. Department of Energy Contract DE-AC06-96RL13200 \\ EDT/ECN: 626252 \\ UC: 620 \\ Org Code: $2 \mathrm{G} 300$ \\ B\&R Code: $39 \mathrm{EW} 40400$ \\ Charge Code: $105559 / \mathrm{A} 000$ \\ Total Pages: 12
}

Key Words: Water Flow Regulator Valves

Abstract: Griswold Tempered Water Flow Regulator Valves Used As Anti-Siphon Valves CGI-SNF-D-47- P4-003

TRADEMARK DISCLAIMER. Reference herein to any specific commercial product, process, or service by trade name, trademark, manufacturer, or otherwise, does not necessarily constitute or imply its endorsement, recommendation, or favoring by the United States Government or any agency thereof or its contractors or subcontractors.

Printed in the United States of America. To obtain copies of this document, contact: Document Control Services, P.O. Box 950, Mailstop H6-08, Richland WA 99352, Phone (509) 372-2420; Fax (509) 376-4989.

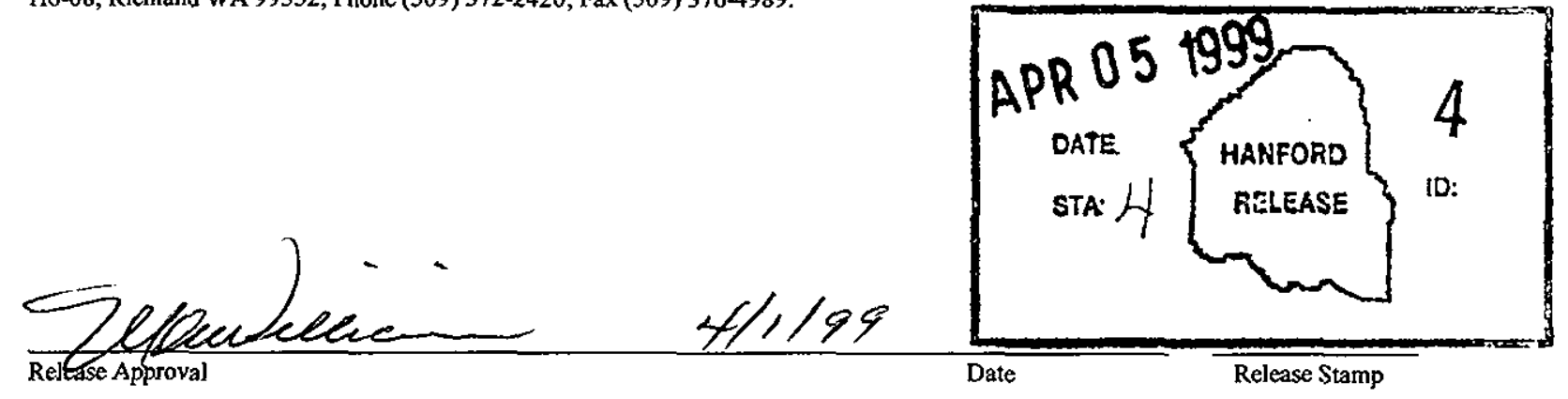


Commercial Grade Item Upgrade Dedication Form ECN No. NA CGI No. CGI-SNF-D-47-P4-003 Title: GRISWOLD TEMPERED WATER FLOW REGULATOR VALVES USED AS ANTI-SIPHON VALVES

\begin{tabular}{|c|c|c|c|}
\hline Item No.: NA & Manufacturer: & Suppl & \\
\hline \multicolumn{2}{|l|}{ Mfg. Part/Model No:: } & \multicolumn{2}{|l|}{ Supplier's PN: } \\
\hline \multicolumn{4}{|l|}{ Part Description: } \\
\hline \multicolumn{4}{|l|}{ End Use Description: } \\
\hline \multicolumn{4}{|c|}{ (6) } \\
\hline $\begin{array}{l}\text { Equipment No:: } \\
\text { FCV-1*22; } \\
\text { FCV-1*23 }\end{array}$ & $\begin{array}{l}\text { Specification No.: W-441- } \\
\text { P4, Rev } 2\end{array}$ & $\begin{array}{l}\text { Manufacturer: Griswold } \\
\text { Controls }\end{array}$ & Past P.O. No.: NA \\
\hline $\begin{array}{l}\text { Manufacturer's Part/ } \\
\text { Model No.: } \mathbf{4 9 0 2 F}\end{array}$ & Equipment Supplier (if dfferer & from manufacturer): TBD & $\begin{array}{l}\text { Equip. Supplier's Part No.: } \\
\text { NA }\end{array}$ \\
\hline \multicolumn{4}{|c|}{$\begin{array}{l}\text { Component Description: FCV -1*22 and } 1 * 23 \text { are Griswold constant flow regulators used as anti-siphon } \\
\text { valves in the tempered water system, they fail closed but valve cartridge orifice allows minimum } \\
\text { flow to prevent loss of water from the MCO/CASK annulus. }\end{array}$} \\
\hline 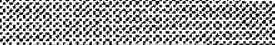 & 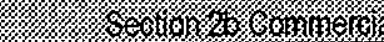 & 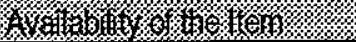 & \\
\hline \multicolumn{4}{|c|}{$\begin{array}{l}\text { 1. Is the Item available from a catalogue from a qualified NQA1 supplier } \\
\text { interface Engineer or BTR)? } \\
\text { [ ] YES (go to \#2 below) } \\
\text { [X] NO (go to procedure step 5.3.2, proceed to dedicate ltem) }\end{array}$} \\
\hline \multicolumn{4}{|c|}{ 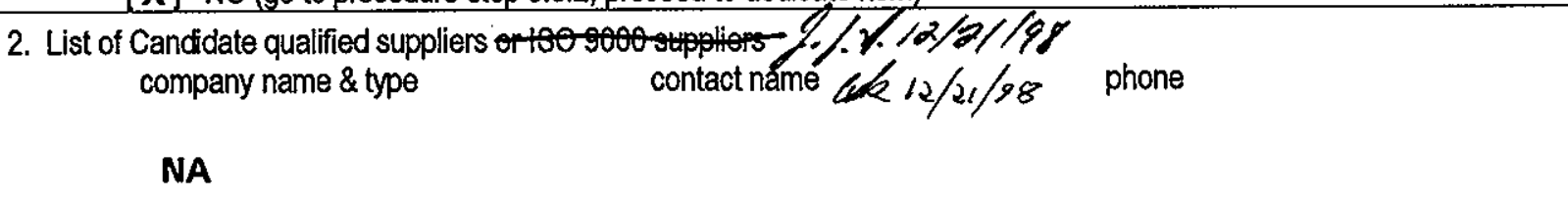 } \\
\hline \multicolumn{4}{|c|}{ 3. Recommended Procurement Strategy(coordinate with project CGI interface Engineer or BTR): NA } \\
\hline 4) & 18: = & 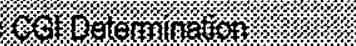 & \\
\hline \multicolumn{4}{|c|}{$\begin{array}{l}\text { 1. Question \#1: Is the Item subject to design or specification requirements that are unique to nuclear facilities or activities? } \\
\text { [ ] YES (the Item is not commercial grade) } \\
\text { [X] NO (continue) }\end{array}$} \\
\hline $\begin{array}{l}\text { 2. Question \#2: is } \\
\text { [1] NO (the } \\
\text { [X] YES (co }\end{array}$ & $\begin{array}{l}\text { cod in applications other than } \mathrm{n} \\
\text { commercial grade) }\end{array}$ & clear facilities or activities? & \\
\hline
\end{tabular}


3. Question \#3: Is the Item ordered from manufacturer/supplier on the basis or specifications set forth in the manufacturers catalog?

[ ] NO (the item is not commercial grade)

$[\mathbf{X}]$ YES (continue)

[X] All three criteria have been satisfied. The ltem meets the definition of commercial grade.

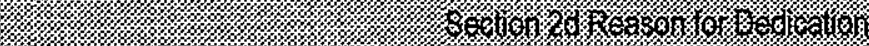

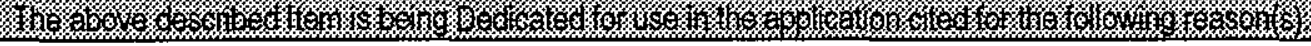

[X] Item is being purchased from a non ESL manufacturer supplier as commercial grade to be used in a Safety Class application.

[ ] Item is being purchased from a non ESL manufacturer supplier as commercial grade to be used in a Safety Significant application.

[ ] Item was purchased from a non ESL manufacturer supplier as commercial grade to be used in a Safety Class application.

[ ] Item was purchased from a non ESL manufacturer supplier as commercial grade to be used in a Safety Significant application.

[ ] Other (like-for-like', similar, substitution, replacement evaluation)

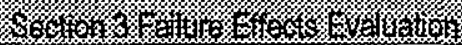

\section{A. Part/Component Safety Function:}

1. The tempered water system's safety function is to prevent overheating the MCO contents and a runaway reaction. The anti-siphon valves contribute to this function by preventing reverse flow siphoning from the MCO annulus.

2.

3.

B. Part/Component Functional Mode:

Safety Function \#1:

[ ] Active - Mechanical or Electrical change of state is required to occur for the component to perform its safety function

[X] Passive - Change of state is not required for the component to perform its safety function

C. Host Component Safety Function (if applicable):

1. NA

2.

3. 
D. Failure Mode(s) and the effects on component or system safety function (see Worksheet 1):

1. Valve Binding - Failure to remain minimally open - Siphon of Annulus Water

2.

3.

4.

5.

8.0.

Environmental Qualification Required:

If yes: Environmental Qualification Requirements

Yes [ ]

No $[\mathbf{X}]$

Limiting Environmental Conditions: Required Safety

Functions:

Qualification Period:

Natural Phenomena Hazard (NPH) Design Required:

If yes: NPH Design Requirements

Performance Category: PC-3

Yes [X]

NPH Design Req'ts.: Seismic Condition A

No [ ]

Required Safety Functions: Prevent siphon of MCO/CASK tempered water

HNF-PRO-97 REV. 0

\section{MCO/CASK tempered water}

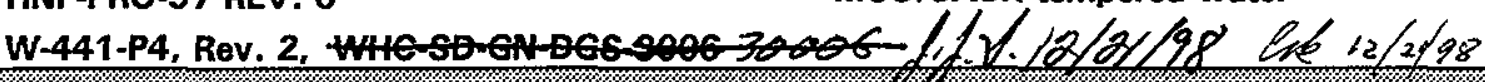

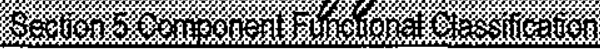

[ X] Safety Class (SC) [ ] General Senice

I ] Safety Significant (SS)

If partcomponent classification is dfferent from host component/system, document basis.

\begin{tabular}{|l|l|l|}
\hline Safety Analysis Report (SAR): HNF- & Drawings: H-1-82161, Rev. 2 \\
ASME B 31.1 & SD-SNF-SAR-002, Rev. 4A & HNF-SD-SNF-SEL-002, Rev. 4 \\
B16 SERIES & & \\
& & \\
& & \\
& & \\
\hline
\end{tabular}

Vendor Manual/Manufacturer/Supplier Information: Griswold F-2723A

Other. NA 


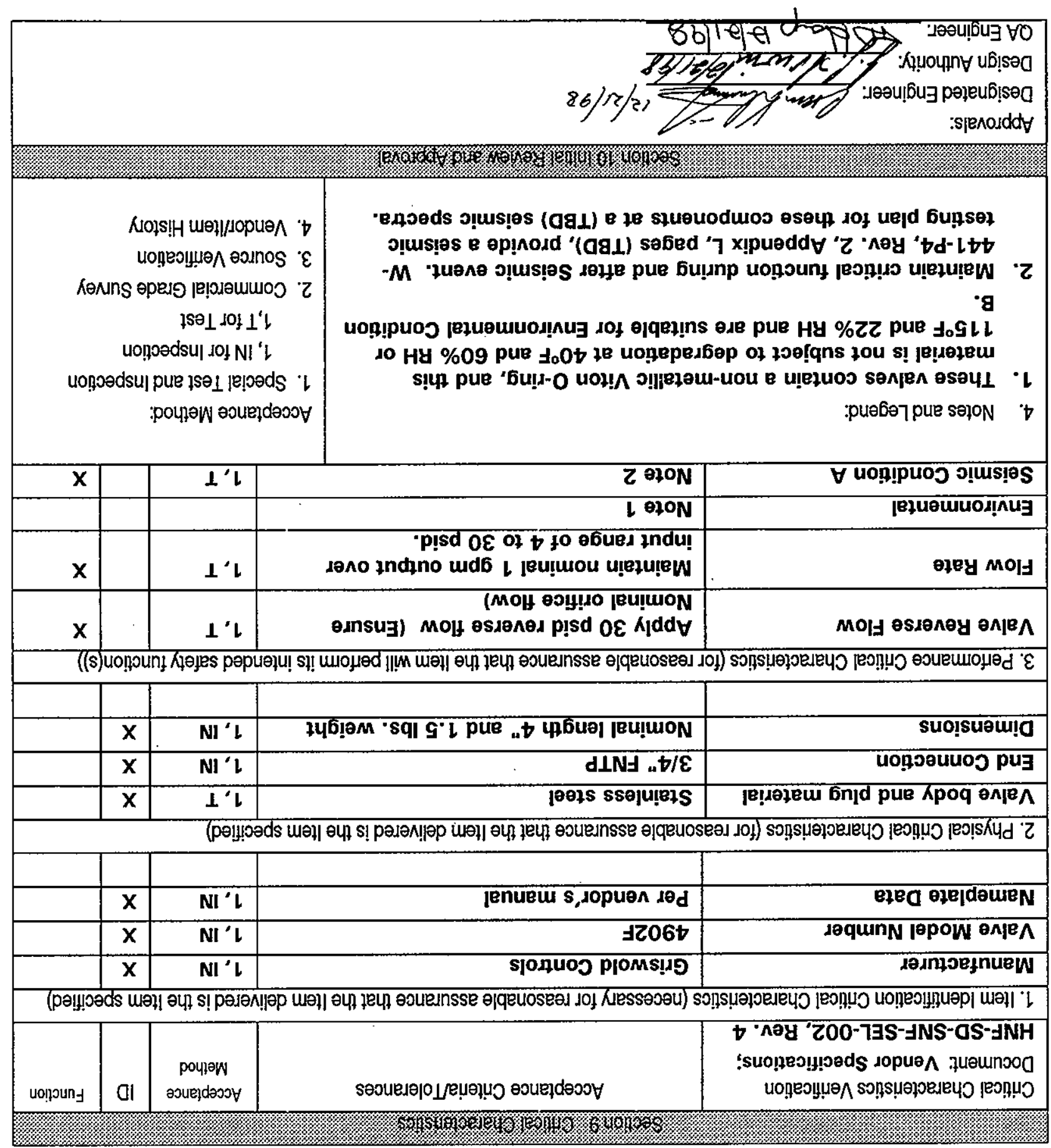

\begin{tabular}{|c|c|}
\hline SLSE JCS & 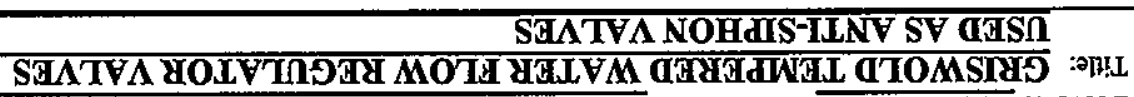 \\
\hline $1130+288 d$ & E00-td-Lt-ם-HNS-IDS ONIOS $\quad$ VN ON NOA \\
\hline $0 . \mathrm{ON}^{\mathrm{N}} \mathrm{N} \cdot \mathrm{Y}$ & นй \\
\hline
\end{tabular}


Commercial Grade Item Upgrade Dedication Form ECN No. NA CGI No. CGI-SNF-D-47-P4-003 Title: GRISWOLD TEMPERED WATER FLOW REGULATOR VALVES USED AS ANTI-SIPHON VALVES

\begin{tabular}{|c|c|c|}
\hline \multicolumn{3}{|c|}{$\begin{array}{c}\text { WORKSHEET } 1 \\
\text { DETERMINATION OF FALURE MECHANISMSMODES }\end{array}$} \\
\hline & \multirow{2}{*}{\multicolumn{2}{|c|}{$\begin{array}{ccc}\text { Applicable to Component } \\
\text { under Evaluation }\end{array}$}} \\
\hline $\begin{array}{l}\text { Typical Failure } \\
\text { Mechanisms }\end{array}$ & & \\
\hline Fracture & $\begin{array}{l}\text { Separation of a solid accompanied by little or no } \\
\text { macroscopic plastic deformation. }\end{array}$ & $\begin{array}{l}\text { Yes [ ] No [X]; if Yes, indicate failure } \\
\text { Mode }\end{array}$ \\
\hline Corrosion & $\begin{array}{l}\text { The gradual deterioration of a material due to } \\
\text { chemical or electrochemical reactions, such as }\end{array}$ & $\begin{array}{l}\text { Yes [ ] No [X]; If Yes, indicate failure } \\
\text { Mode_ }\end{array}$ \\
\hline Erosion & $\begin{array}{l}\text { oxidation, between the material and its environment. } \\
\text { Destruction of materials by the abrasive action of } \\
\text { moving fluids, usually accelerated by the presence } \\
\text { of solid particles carried with the fluid. }\end{array}$ & $\begin{array}{l}\text { Yes [ ] No [X]; If Yes, indicate failure } \\
\text { Mode }\end{array}$ \\
\hline Open Circuit & $\begin{array}{l}\text { An electrical circuit that is unintentionally broken so } \\
\text { that there is no complete path for current flow. }\end{array}$ & $\begin{array}{l}\text { Yes [ ] No [X]; if Yes, indicate failure } \\
\text { Mode }\end{array}$ \\
\hline Short Circuit & $\begin{array}{l}\text { An abnormal connection by which an electrical } \\
\text { current is connected to ground, or to some }\end{array}$ & $\begin{array}{l}\text { Yes [ ] No [X]; If Yes, indicate failure } \\
\text { Mode }\end{array}$ \\
\hline Blockage & $\begin{array}{l}\text { conducting body, resulting in excessiva curtent flow. } \\
\text { Clogging of a filtering medium resulting in the } \\
\text { inability to perform its purification function or }\end{array}$ & $\begin{array}{l}\text { Yes [ ] No [X]; If Yes, indicate failure } \\
\text { Mode }\end{array}$ \\
\hline Seizure & $\begin{array}{l}\text { Binding of a normally moving item through excessive } \\
\text { pressure, temperature, friction, jamming. }\end{array}$ & $\begin{array}{l}\text { Yes [X] No [ ]; If Yes, indicate failure } \\
\text { Mode Valve Binding - Failure to remain }\end{array}$ \\
\hline & & open \\
\hline $\begin{array}{l}\text { Unacceptable } \\
\text { Vibration }\end{array}$ & $\begin{array}{l}\text { Mechanical oscillations produced are beyond the } \\
\text { defined permissible limits due to unbalancing, poor }\end{array}$ & $\begin{array}{l}\text { Yes [ ] No [X]; If Yes, indicate failure } \\
\text { Mode }\end{array}$ \\
\hline Loss of Properties & $\begin{array}{l}\text { support, or rotation at critical speeds. } \\
\text { A loss of mechanical and physical properties of a } \\
\text { material due to exposure to high temperatures, } \\
\text { radiation exposure. }\end{array}$ & $\begin{array}{l}\text { Yes [ ] No [X]; if Yes, indicate failure } \\
\text { Mode }\end{array}$ \\
\hline Excess Strain & $\begin{array}{l}\text { Under the action of excessive external forces the } \\
\text { material of the part has been deformed or distorted. }\end{array}$ & $\begin{array}{l}\text { Yes [ ] No [X]; if Yes, indicate failure } \\
\text { Mode }\end{array}$ \\
\hline Mechanical Creep & $\begin{array}{l}\text { From prolonged exposure to high temperature and } \\
\text { stress, the object will show a slow change in its }\end{array}$ & $\begin{array}{l}\text { Yes [ ] No [X]; If Yes, indicate failure } \\
\text { Mode }\end{array}$ \\
\hline & characteristics. & \\
\hline Ductile Fracture & $\begin{array}{l}\text { Fracture characterized by tearing of metal } \\
\text { accompanied by appreciable gross plastic } \\
\text { deformation. }\end{array}$ & No $[X]$; if Yes, indicate failure \\
\hline & E & 10. \\
\hline 1. & & \\
\hline 2. & & \\
\hline 3. & & \\
\hline 4. & & \\
\hline 5. & & \\
\hline
\end{tabular}


Title: GRISWOLD TEMPERED WATER FLOW REGULATOR VALVES USED AS ANTI-SIPHON VALVES

\section{CHECKLIST 1}

\section{ACCEPTANCE METHOD 1}

SPECIAL TEST/ANSPECTION VERIFICATION

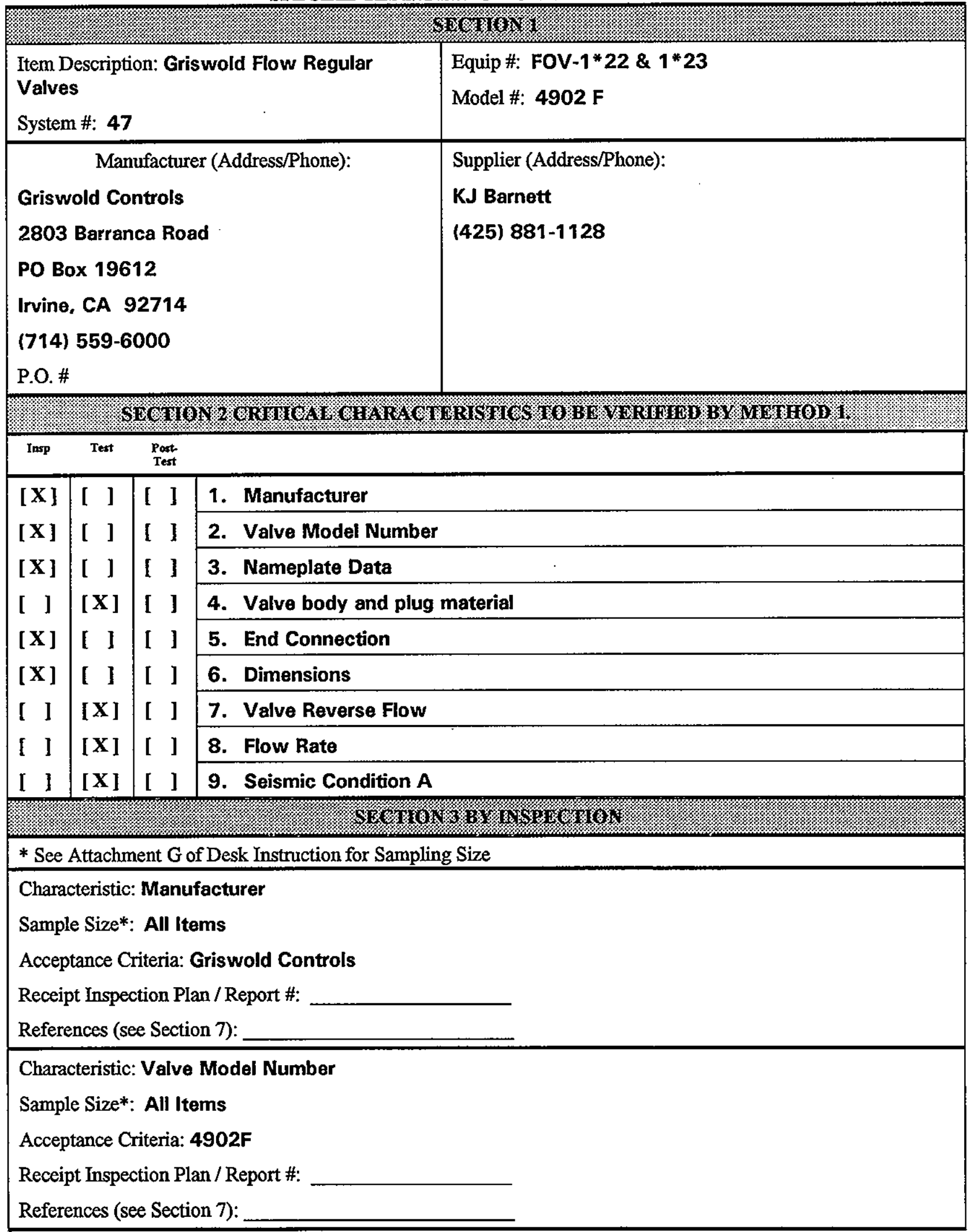




\section{Characteristic: Nameplate Data}

Sample Size*: All Items

Acceptance Criteria: Per vendor's manual

Receipt Inspection Plan / Report \#:

References (see Section 7): Vendor's Sheet F-2723A

Characteristic: End Connection

Sample Size*: All Items

Acceptance Criteria: 3/4" FNTP

Receipt Inspection Plan / Report \#:

References (see Section 7):

Characteristic: Dimensions

Sample Size*: All Items

Acceptance Criteria: Nominal length $4^{n}$ and 1.5 lbs. weight

Receipt Inspection Plan / Report \#:

References (see Section 7):

\section{"}

* See Attachment G of Desk Instruction for Sampling Size

Test To Be Performed by:

Number of Items to be Tested:

[ ] Purchaser

[ ] Supplier/Manufacturer**

Test/Inspection Location:

[ ] Other

Characteristic for Test: Valve body and plug materiai

Acceptance Criteria: Stainless steel

Sample Size*: Normal Sampling Size

Actual Test Value:

Test Plan and Report \#:

References (see Section 7):

Characteristic for Test: Valve Reverse Flow

Acceptance Criteria: Apply 30 psid reverse flow (Ensure Nominal orifice flow)

Sample Size*: Normal Sampling Size

Actual Test Value:

Test Plan and Report \#: References (see Section 7):

Characteristic for Test: Flow Rate

Acceptance Criteria: Maintain nominal $1 \mathrm{gpm}$ output over input range of 4 to 30 psid.

Sample Size*: Normal Sampling Size

Actual Test Value:

Test Plan and Report \#: References (see Section 7): 
Characteristic for Test: Seismic Condition A

Acceptance Criteria: Maintain critical function during and after Seismic event.

Sample Size*: Normal Sampling Size

Actual Test Value:

Test Plan and Report \#: References (see Section 7):

**If Supplier/Manufacturer or Other, Refer to CGI Checklist-2 for Support Information 
Commercial Grade Item Upgrade Dedication Form ECN No. NA CGI No. CGI-SNF-D-47-P4-003 Title: GRISWOLD TEMPERED WATER FLOW REGULATOR VALVES USED AS ANTI-SIPHON VALVES

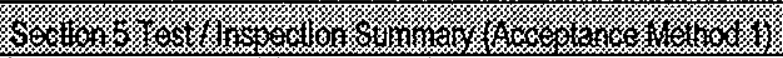

1. SUMMARY OF VERIFIED CRITICAL CHARACTERISTICS, THEIR VERIFICATION METHODS, AND RESULTS

ITEM DESCRIPTION:

\begin{tabular}{|c|c|c|c|c|c|c|c|c|c|c|c|}
\hline \multicolumn{4}{|c|}{ Critical Characteristics } & \multicolumn{8}{|c|}{ Verification Results } \\
\hline Critical Characteristics & Acceptance Criteria/Tolerances & $\mathrm{m}$ & Function & $\begin{array}{l}\text { Method } \\
\text { T/IN }\end{array}$ & $\begin{array}{l}\text { Procedure } \\
\text { or RR\# }\end{array}$ & $\begin{array}{l}\text { Check- } \\
\text { list ID }\end{array}$ & $\begin{array}{c}\text { Number } \\
\text { Tested }\end{array}$ & $\begin{array}{l}\text { Number } \\
\text { Failed }\end{array}$ & $\begin{array}{l}\text { Verifying } \\
\text { Organization }\end{array}$ & Printed Name Signature & Date \\
\hline Manufacturer & Griswold Controls & $\mathbf{X}$ & & & & & & & & & \\
\hline Valve Model Number & $4902 F$ & $\mathbf{X}$ & & & & & & & & & \\
\hline Nameplate Data & Per vendor's manual & $\mathbf{X}$ & & & & & & & & & . \\
\hline $\begin{array}{l}\text { Valve body and plug } \\
\text { material }\end{array}$ & Stainless steel & $\mathbf{X}$ & & & & & & & & & \\
\hline End Connection & 3/4" FNTP & $\mathbf{X}$ & & & & & & & & & \\
\hline Dimensions & $\begin{array}{l}\text { Nominal length } 4 " \text { and } \\
1.5 \mathrm{lbs} \text {. weight }\end{array}$ & $\mathbf{X}$ & & & & & & & & & \\
\hline Valve Reverse Flow & $\begin{array}{l}\text { Apply } 30 \text { psid reverse } \\
\text { flow (Ensure Nominal } \\
\text { orifice flow) }\end{array}$ & & $\mathbf{X}$ & & & & & & & & \\
\hline Flow Rate & $\begin{array}{l}\text { Maintain nominal } 1 \mathrm{gpm} \\
\text { output over input range } \\
\text { of } 4 \text { to } 30 \text { psid. }\end{array}$ & & $\mathbf{X}$ & & & & & & & & \\
\hline Environmental & NA & & $\mathbf{X}$ & & & & & & & & \\
\hline Seismic Condition A & Maintain critical function & & $\mathbf{X}$ & & & & & & & & \\
\hline
\end{tabular}


Commercial Grade Item Upgrade Dedication Form

ECN No. NA CGI No. CGI-SNF-D-47-P4-003

Title: GRISWOLD TEMPERED WATER FLOW REGULATOR VALVES USED AS ANTI-SIPHON VALVES
Rev. No. 0

Page 10 of 11

SNF 3878

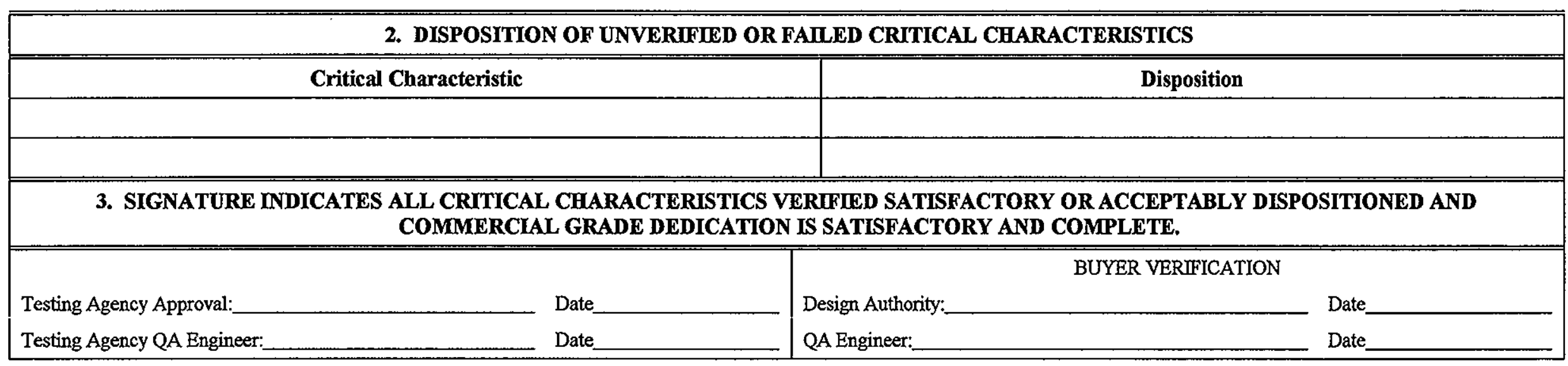




\begin{tabular}{|c|c|}
\hline Name & \\
\hline Design Authority & ( \\
\hline QA & ( \\
\hline QC & ( \\
\hline Cog - Engineer & ( \\
\hline CGI Engineer & ( \\
\hline Procurement Engineer & ( \\
\hline \multicolumn{2}{|c|}{ Other $\quad\left(\begin{array}{ll}1 \\
0\end{array}\right.$} \\
\hline \multicolumn{2}{|c|}{ 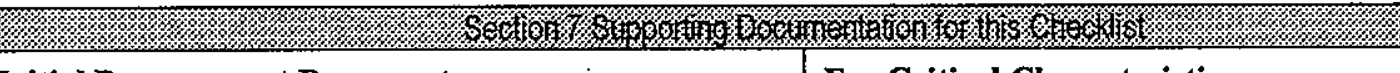 } \\
\hline Initial Procurement Documents & For Critical Characteristics \\
\hline \multicolumn{2}{|l|}{ [ ] Drawings: } \\
\hline \multicolumn{2}{|l|}{ [ ] Manuals (specify type \& number): } \\
\hline \multicolumn{2}{|l|}{ [ ] Design Calculations } \\
\hline \multicolumn{2}{|l|}{ [ ] Installation Instructions } \\
\hline \multicolumn{2}{|l|}{ [ ] Operation Instructions } \\
\hline \multicolumn{2}{|l|}{ [ ] Calibration Instructions } \\
\hline \multicolumn{2}{|l|}{ [ ] Manufacturer's Recommended Spare Parts List } \\
\hline \multicolumn{2}{|l|}{ [ ] Other: } \\
\hline \multicolumn{2}{|l|}{ Procurement Documents } \\
\hline \multicolumn{2}{|l|}{ [ ] Certificate of Conformance/Compliance } \\
\hline \multicolumn{2}{|l|}{ [ ] Seismic Qualification Certificate } \\
\hline \multicolumn{2}{|l|}{ [ ] Environmental Qualification Certificate } \\
\hline \multicolumn{2}{|l|}{ [ ] Test Report (s): } \\
\hline \multicolumn{2}{|l|}{ [ ] Inspection Report (s): } \\
\hline \multicolumn{2}{|l|}{ [ ] CMTRs for ASME Pressure Retaining Materials } \\
\hline \multicolumn{2}{|l|}{ I I Valve Seat Leakage Report } \\
\hline \multicolumn{2}{|l|}{ [ ] Weld Records } \\
\hline \multicolumn{2}{|l|}{ [ ] Material Traceability Record } \\
\hline [ ] Other: & \\
\hline
\end{tabular}

